\title{
Unusual location of the geotail magnetopause near lunar orbit: a case study
}

\author{
W. S. Shang, B. B. Tang, Q. Q. Shi, A. M. Tian, X.-Y. Zhou, \\ Z. H. Yao, A. W. Degeling, I. J. Rae, S. Y. Fu, J. Y. Lu, \\ Z. Y. Pu, A. N. Fazakerley, M. W. Dunlop, G. Facskó, \\ J. Liu and M. Wang
}

\section{Published version information}

Citation: WS Shang et al. "Unusual location of the geotail magnetopause near lunar orbit: a case study." Journal of Geophysical Research: Space Physics, vol. 125, no. 4 (2020): e2019JA027401.

DOI: $\underline{10.1029 / 2019 J A 027401}$

This version is made available in accordance with publisher policies. Please cite only the published version using the reference above. This is the citation assigned by the publisher at the time of issuing the APV. Please check the publisher's website for any updates. 


\section{JGR Space Physics}

\section{RESEARCH ARTICLE \\ 10.1029/2019JA027401 \\ Unusual Location of the Geotail Magnetopause Near Lunar Orbit: A Case Study}

Key Points:

- The large-scale deflection of the magnetopause was observed by ARTMIES probes at lunar distance near midnight (the full moon time)

- The magnetopause deflection is due to the influence of the solar wind $V_{Y \text {-windsock effect }}$

- This case study provides a new point of view of the interaction of the solar wind and lunar nearside surface at the full moon time

Correspondence to:

Q. Q. Shi,

sqq@sdu.edu.cn

Citation:

Shang, W. S., Tang, B. B., Shi, Q. Q., Tian, A. M., Zhou, X.-Y., Yao, Z. H., et al. (2020). Unusual location of the geotail magnetopause near lunar orbit: A case study. Journal of Geophysical Research: Space Physics, 125, e2019JA027401. https://doi.org/ 10.1029/2019JA027401

Received 12 SEP 2019

Accepted 21 MAR 2020

Accepted article online 14 APR 2020

(C)2020. American Geophysical Union. All Rights Reserved.

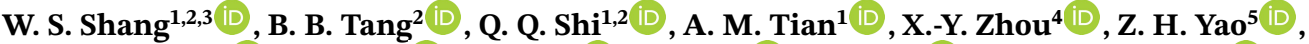

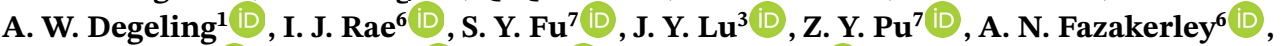

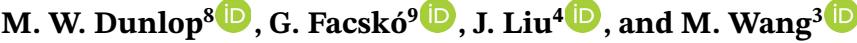 \\ ${ }^{1}$ Shandong Provincial Key Laboratory of Optical Astronomy and Solar-Terrestrial Environment, School of Space Science \\ and Physics, Institute of Space Sciences, Shandong University, Weihai, China, ${ }^{2}$ State Key Laboratory of Space Weather, \\ Center for Space and Applied Research, Chinese Academy of Sciences, Beijing, China, ${ }^{3}$ Institute of Space Weather, \\ Nanjing University of Information Science \& Technology, Nanjing, China, ${ }^{4}$ Earth, Planetary, and Space Sciences, \\ UCLA, Los Angeles, CA, USA, ${ }^{5}$ Laboratoire de Physique Atmosphérique et Planétaire, STAR institute, Université de \\ Liège, Liège, Belgium, ${ }^{6}$ UCL Mullard Space Science Laboratory, Dorking, RH5 6NT, UK, ${ }^{7}$ School of Earth and Space \\ Sciences, Peking University, Beijing, China, ${ }^{8}$ Space Sciences Division, SSTD, Rutherford Appleton Laboratory, \\ Oxfordshire, UK, ${ }^{9}$ Rhea System GmbH, Darmstadt, Germany
}

\begin{abstract}
The Earth's magnetopause is highly variable in location and shape and is modulated by solar wind conditions. On 8 March 2012, the ARTEMIS probes were located near the tail current sheet when an interplanetary shock arrived under northward interplanetary magnetic field conditions and recorded an abrupt tail compression at $\sim(-60,0,-5) R_{E}$ in Geocentric Solar Ecliptic coordinate in the deep magnetotail. Approximately 10 minutes later, the probes crossed the magnetopause many times within an hour after the oblique interplanetary shock passed by. The solar wind velocity vector downstream from the shock was not directed along the Sun-Earth line but had a significant $Y$ component. We propose that the compressed tail was pushed aside by the appreciable solar wind flow in the $Y$ direction. Using a virtual spacecraft in a global magnetohydrodynamic (MHD) simulation, we reproduce the sequence of magnetopause crossings in the $X$ - $Y$ plane observed by ARTEMIS under oblique shock conditions, demonstrating that the compressed magnetopause is sharply deflected at lunar distances in response to the shock and solar wind $V_{Y}$ effects. The results from two different global MHD simulations show that the shocked magnetotail at lunar distances is mainly controlled by the solar wind direction with a timescale of about a quarter hour, which appears to be consistent with the windsock effect. The results also provide some references for investigating interactions between the solar wind/magnetosheath and lunar nearside surface during full moon time intervals, which should not happen in general.
\end{abstract}

\section{Introduction}

The magnetopause is a boundary that separates the magnetospheric plasma from the solar wind. This region plays an important role in physics of solar wind-magnetosphere coupling. The concept of the magnetopause and fundamental magnetopause theory were first presented by Chapman and Ferraro (1931). The magnetopause has been observed by satellites since 1960s, and many empirical models have been proposed and magnetohydrodynamic (MHD) simulations have been conducted regarding its shape in the dayside and near tail regions (e.g., Liu et al., 2015; Lu et al., 2011; Palmroth et al., 2001; Shue et al., 1997, 1998). However, most studies have only investigated the effect of interplanetary magnetic field (IMF) $B_{Z}$ and the solar wind dynamic pressure (Dp) in the dayside and near tail regions (e.g., Fairfield, 1971; Lu et al., 2013; Sibeck et al., 1991; Shue et al., 1997). The waves, vortexes, burst bulk flows, and substorms excited by magnetopause changes/solar wind dynamic pressure pulses were studied by single and multiple spacecraft (e.g., Shi et al., 2013; Xiao et al., 2010; Yao et al., 2017; Zong et al., 1997, 2009, 2012; Zhou et al., 2009, 2013; Zhang et al., 2010). During southward IMF conditions, dayside magnetic reconnection transfers interplanetary magnetic field to magnetosphere and stores it in magnetotail (Coroniti \& Kennel, 1972). Under northward IMF conditions, however, the reconnected magnetic flux from high-latitude reconnection sinks into the magnetopause forming a boundary layer (Shi et al., 2009, 2013; Song \& Russell, 1992). In addition to the above factors, the cone angle (the angle between the direction of the IMF and Sun-Earth line) (Fairfield et al., 1990; Park et al., 
2016) and Earth's dipole tilt angle (Lu et al., 2011; Nowada et al., 2009) also play an important role in determining the location and shape of the magnetopause in the dayside and near Earth regions. Those regions were widely studied using multipoint measurements and MHD simulations (e.g., Lu et al., 2011; Palmroth et al., 2001; Pu et al., 2005; Shue et al., 1997, 1998; Shi et al., 2005, 2006, 2019).

The first investigations of mid and distant magnetotail were conducted using spacecraft observations in the late 1960s. Ness et al. (1967) demonstrated that the magnetopause was highly variable in response to both IMF and solar wind variations in the distant tail beyond the lunar orbit by Explorer 33. Later, Hardy et al. (1979) proposed that an observed dawn-dusk asymmetry in the magnetopause shape also resulted from nonzero IMF $B_{Y}$ at lunar distance, based on Apollo mission data. Recently, many studies have employed global MHD models to study the effect of the IMF in the cross-section of the magnetotail at lunar distance. Their results confirmed that the twisting of magnetopause results from nonzero IMF $B_{Y}$ (e.g., Facskó et al., 2016; Gordeev et al., 2013; Sibeck \& Lin, 2014; Vörös et al., 2014; Wang et al., 2014). They also demonstrated that the magnetopause could elongate further along the direction of IMF in the distant magnetotail than in the near-Earth regions. Statistical results from ARTEMIS mission (Angelopoulos, 2011) observations also confirmed that both IMF $B_{Y}$ and IMF $B_{Z}$ have important effects on the location and shape of the magnetotail at lunar distance (Akay et al., 2019; Liu et al., 2016). Several studies also show a relationship between magnetopause location and solar wind flow vector changes (the effect of solar wind $V_{Y}$ and $V_{Z}$ ). Using simultaneous observations from Explorers 33 and 35, Howe et al. (1972) first recognized that the magnetotail axis lies aligned with the direction of the solar wind velocity, which results in north/south and east/west flapping motions in response to variations in the direction of the solar wind velocity. Based on Geotail observations, Shodhan et al. (1996) proposed that the windsock mechanism (the solar wind flow vector moving away from the aberrated Sun-Earth line) caused flapping of the magnetotail, while intrinsic expansions and contractions of the magnetotail are in response to substorms (the breathing mechanism) beyond $150 R_{E}$ downtail. In addition, the case mentioned by Shodhan et al. (1996) also showed that the windsock caused variations on a timescale of hours, and breathing dominates on a timescale of tens of minutes. The flapping motions caused by solar wind direction changes and shock/corotating interaction regions (Opitz et al., 2014) in the deeper tail (at X= -230 $R_{E}$ ) were confirmed by Grygorov et al. (2014). Due to small fluctuations of the solar wind velocity direction, Grygorov et al. (2014) found that the Wind satellite entered the south lobe and then scanned the whole magnetotail in $Z$ direction before the shock arrival. Then, the Wind satellite returned to the south lobe region, which was responded to the more/stronger northward solar wind flows in the shock downstream. It was confirmed that the response of the geomagnetic tail to the varying solar wind velocity direction could be the cause of the multiple magnetopause crossings in the distant tail. However, the magnetopause is more flexible in the distant tail than that at lunar distance (Akay et al., 2019). The amplitudes of these flapping motions responding to the variations in the direction of the solar wind velocity become larger with downtail distance (Akay et al., 2019; Howe et al., 1972; Sun et al., 2010). As mentioned above, the location and shape of magnetopause can be affected by the interplanetary and solar wind conditions both in the near-Earth region and in the distant magnetotail. However, it is unclear how the magnetopause location and structure react to extreme solar wind conditions at lunar distance.

Each lunar month, the Moon typically enters the terrestrial magnetotail for several days around full moon and is exposed to the solar wind or magnetosheath for the remainder of its orbit. Within the magnetotail, the Moon is exposed to the hot plasma of the terrestrial plasma sheet (e.g., Harada et al., 2012, 2014; Kallio \& Facskó, 2014). Plasma parameters in the magnetosphere significantly differ from those in both the solar wind and magnetosheath. Therefore, the lunar space environment can be expected to become altered by its exposure to different conditions (e.g., Saito et al., 2008; Wang et al., 2017, 2018). It is critical to distinguish the relative location of the Moon for real-time observations of key particle species on the lunar surface, especially hydroxyl or water molecules (Wang et al., 2018).

The Acceleration, Reconnection, Turbulence, and Electrodynamics of the Moon's Interaction with the Sun (ARTEMIS) mission (Angelopoulos, 2011; Sibeck et al., 2011) provides us a good opportunity to investigate the location and shape of the magnetopause at lunar distance. We will show in this paper that the windsock effect can lead to a large-scale deflection of the magnetopause at lunar orbit ( $\sim 60 R_{E}$ downtail), where the ARTEMIS probe encountered magnetosheath-like plasma flows at the full moon time. We also investigate the big picture of the shocked magnetosphere at lunar distance using the piecewise parabolic method (PPM) global MHD model (Hu et al., 2007). We present in Section 2 the ARTEMIS data and its measurements as well as the interplanetary solar wind data. In Section 3, we describe an overview of magnetopause crossing 
events during the rapid flapping of the magnetosphere under the extreme solar wind and strongly northward IMF conditions. In Section 4, we reproduce the magnetopause crossing by the global MHD simulations at lunar distance. Comparing and contrasting the results between observations and simulations in Section 5, we discuss how the magnetopause at lunar distance responds to the solar wind conditions.

\section{Data}

In this study, we use ion and magnetic field data from the two probes (P1 and P2) of the ARTEMIS mission (Angelopoulos, 2011; Sibeck et al., 2011). The ARTEMIS dual probes were raised in apogee from the five Time History of Events and Macroscale Interactions during Substorms (THEMIS) spacecraft in the near-Earth magnetosphere such that they were captured into lunar orbit in early 2010. The dual probes are equipped with fluxgate magnetometer (FGM) instruments (Auster et al., 2008) to provide magnetic field data and electrostatic analyzer (ESA) instruments (McFadden et al., 2008) to provide energy-resolved ion ( $5 \mathrm{eV}$ to $25 \mathrm{KeV}$ ) data. Solar wind and IMF data are obtained from the Wind satellite (Farrell et al., 1995; Gloeckler et al., 1995), ACE satellite (King \& Papitashvili, 2005), and four Cluster satellites (Balogh et al., 2001; Reme et al., 2001). On 8 March 2012, the ARTEMIS probes simultaneously observed high-density $\left(\sim 10 \mathrm{~cm}^{-3}\right)$, high-speed $(\sim-600 \mathrm{~km} / \mathrm{s})$ magnetosheath-like flows many times within 1 hour. The ARTEMIS probes were near and above the neutral sheet at the full moon time in the midnight region $\left(Y=0 R_{E}, Z=-5.4\right.$ $R_{E}$ ) before the first outward magnetopause crossing. Geocentric solar ecliptic (GSE) coordinates are used throughout this study.

\section{Observations}

Using Wind, ACE, and Cluster-1 probes, we investigate the plasma and magnetic field parameters in interplanetary space as shown in Figure 1. Figures 1a to 1f show the interplanetary magnetic field vector, magnetic field strength, proton density, three components of the solar wind velocity, dynamic pressure, and energy flux measured by Wind probe at $\sim(209,93,6) R_{E}$, respectively. These plots show that, prior to the arrival of shock at the location of Wind ( 10:30 UT, blue dashed line in the left-hand plots), the IMF $B_{Z}$ component was dominant, the IMF $B_{Y}$ component was close to $0 \mathrm{nT}$, the solar wind velocity was $\sim(-540,0,-50) \mathrm{km} / \mathrm{s}$, and the dynamic pressure was about $3 \mathrm{nPa}$. Upon the shock arrival (after $\sim 10: 30$ UT), the $X$ component of solar wind $\left(V_{X}\right)$ jumped from $\sim-540$ to $\sim-750 \mathrm{~km} / \mathrm{s}, V_{Y}$ turned further toward dawn from $\sim 0$ to $\sim-250 \mathrm{~km} / \mathrm{s}$, and $V_{Z}$ also changed from $\sim-50$ to $\sim-120 \mathrm{~km} / \mathrm{s}$. Meanwhile, the dynamic pressure and magnetic field strength increased to $15 \mathrm{nPa}$ and $15 \mathrm{nT}$, respectively.

It is worth noting that the magnetic field strength jumped from $\sim 8$ to $30+\mathrm{nT}$ at $\sim 10: 44$ UT measured by ACE probe at $\sim(236,-35,-18) R_{E}$, marked by the red dashed line in Figures $1 \mathrm{~h}-1 \mathrm{k}$, the location of the ACE probe was farther away from the Earth (i.e., closer to the Sun) than the Wind probe. However, the ACE probe encountered the shock later than Wind probe. The shock was also seen by Cluster-1 probe, located at $\sim(16,1,-12) R_{E}$, at $\sim 11: 02 \mathrm{UT}$, which is marked by the black dashed line in Figures 1h-1k. Based on the assumption of plane wave propagation in interplanetary space, we conclude that the shock was extremely oblique as it propagated toward the Earth with a speed of $\sim 620 \mathrm{~km} / \mathrm{s}$ in the $X-Y$ plane. We also take the four Cluster satellites' magnetic field parameters into account to investigate the shock by the timing method. The results are that the speed and normal of the shock are about $V_{\text {shock }}=653 \mathrm{~km} / \mathrm{s}$ and $N_{\text {shock }}=(-0.75,-0.47,-0.46)$, respectively. Thus, we can infer that the shock impacted the magnetosphere slightly above and duskward of the subsolar point ("shock-magnetopause tangent" region) at 11:00 UT, and subsequently propagated downtail to the vicinity of lunar orbit and the ARTEMIS probes location by 11:10 UT.

Figure 2 presents the magnetic field and plasma observations of the ARTEMIS P1 and P2 probes from 11:00 UT to 11:30 UT. The magnetic field data are taken from FGM instruments, whereas the plasma data are from ESA instruments. Inside the magnetosphere, $B_{X}=\sim 4 \mathrm{nT}, B_{Y}=B_{Z}=\sim 0 \mathrm{nT}$, indicating that the ARTEMIS probes were near and above the neutral sheet in the midnight region prior to the arrival of the shock at 11:10 UT. Meanwhile, solar wind conditions upstream of the shock in the vicinity of lunar orbit (inferred from the solar wind monitoring shown in Figure 1) are relatively quiet.

The blue shaded area in the figure starts at the (inferred) moment of the shock arrival, corresponds to an interval when there were two neutral sheet (NS) crossings by the spacecraft, shown by all parameters. These two crossings indicate dynamic changes occurring in the geotail magnetosphere in response to the shock 

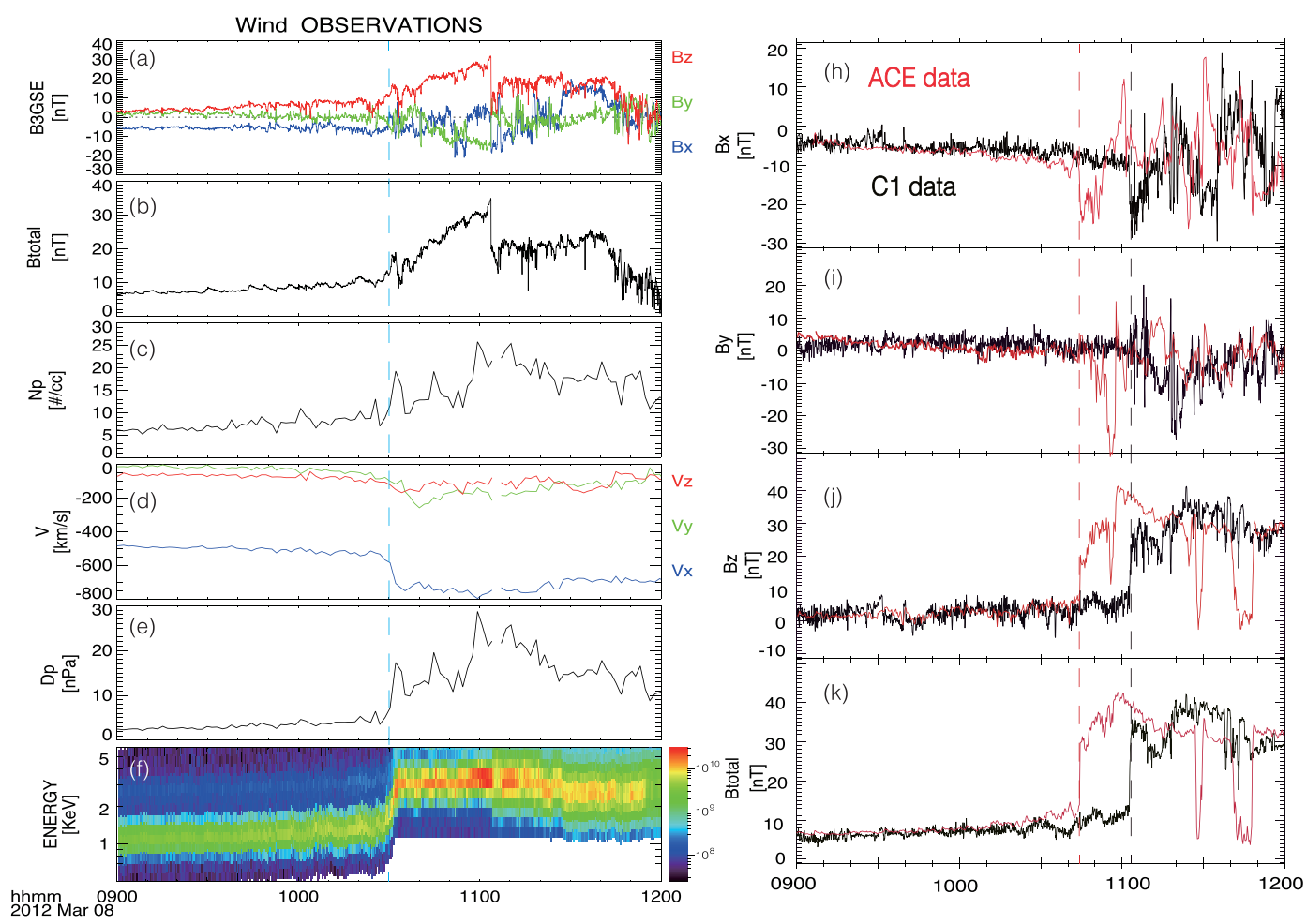

Figure 1. The (a) components of the interplanetary magnetic field (IMF), (b) the magnitude of the IMF, (c) solar wind density, (d) three components of the solar wind velocity, (e) dynamic pressure, and (f) energy fluxes measured by Wind probe and (h) $\operatorname{IMF} B_{X}$, (i) $\operatorname{IMF} B_{Y}$, (j) IMFB $B_{Z}$, and (k) interplanetary magnetic field strength observed by ACE and Cluster-1 on March 08, 2012. The dashed lines marked by blue, red, and black colors corresponding to the sudden commencement time of the shock measured by Wind, ACE, and Cluster-1 probes, respectively.

passage. During the blue shaded interval, a dawnward plasma flow with -300 km/s was seen by the $\mathrm{P} 1$ and P2 probes, and the ion density reached $\sim 2 \mathrm{~cm}^{-3}$ in the NS.

During the subsequent time interval from 11:15 UT to 11:20 UT, the ARTEMIS probes encountered tailward high-speed flows accompanied by an increase in $B_{X}$ and an increase in total magnetic field strength from $\sim 0$ $\mathrm{nT}$ to $\sim 40 \mathrm{nT}$, indicating that the probes have moved to the lobe region (Baumjohann et al., 1990; Pan et al., 2015, 2016; Shang et al., 2014; Wei et al., 2014; Yao et al., 2017). The plasma density in the lobe region is shown to have increased during this interval (compared to the interval shown prior to shock arrival, reaching $1 \mathrm{~cm}^{-3}$ ), presumably due to compression of the geotail plasma following the shock (Gou et al., 2016).

The green shaded region in the figure corresponds to an interval during which the spacecraft undergo multiple crossings of the magnetopause boundary, where magnetosheath-like flows are characterized by $V_{s w}=\sim-600 \mathrm{~km} / \mathrm{s}$, an abrupt change in $B_{X}$ and $B_{Z}$ (indicating the existence of significant current), and enhanced density and thermal pressure compared to the magnetospheric lobe plasma. Figure 2 shows that the probes were both in the magntosheath region during $~ 11: 20$ UT to $\sim 11: 25: 30$ UT and that probe P1 briefly reenters the magnetosphere on two occasions between 11:23 UT and 11:25 UT, before both probes reenter the magnetosphere at $\sim 11: 26 \mathrm{UT}$.

Figure 1 shows that IMF $B_{Z}$ is the dominant component and IMF $B_{Y} \sim 0 \mathrm{nT}$ in the downstream plasma measured immediately after the arrival of the shock; however, IMF $B_{Y}$ becomes increasingly significant after 10-15 minutes. Therefore, for the sake of simplicity, we restrict our attention to the interval up to 15 minutes after the shock arrival in the ARTEMIS data ( 11:25 UT), in order to exclude the effect of IMF $B_{Y}$ in the magnetotail (Akay et al., 2019; Sibeck \& Lin, 2014; Wang et al., 2014) in this study.

During times when the moon is full, the ARTEMIS probes are located very close to the local midnight sector with $X_{G S E}=\sim-60 R_{E}$, which normally corresponds to the deep magnetotail. It is of interest to consider how the actions of the shock and solar wind deform the magnetosphere during this particular event, enabling 


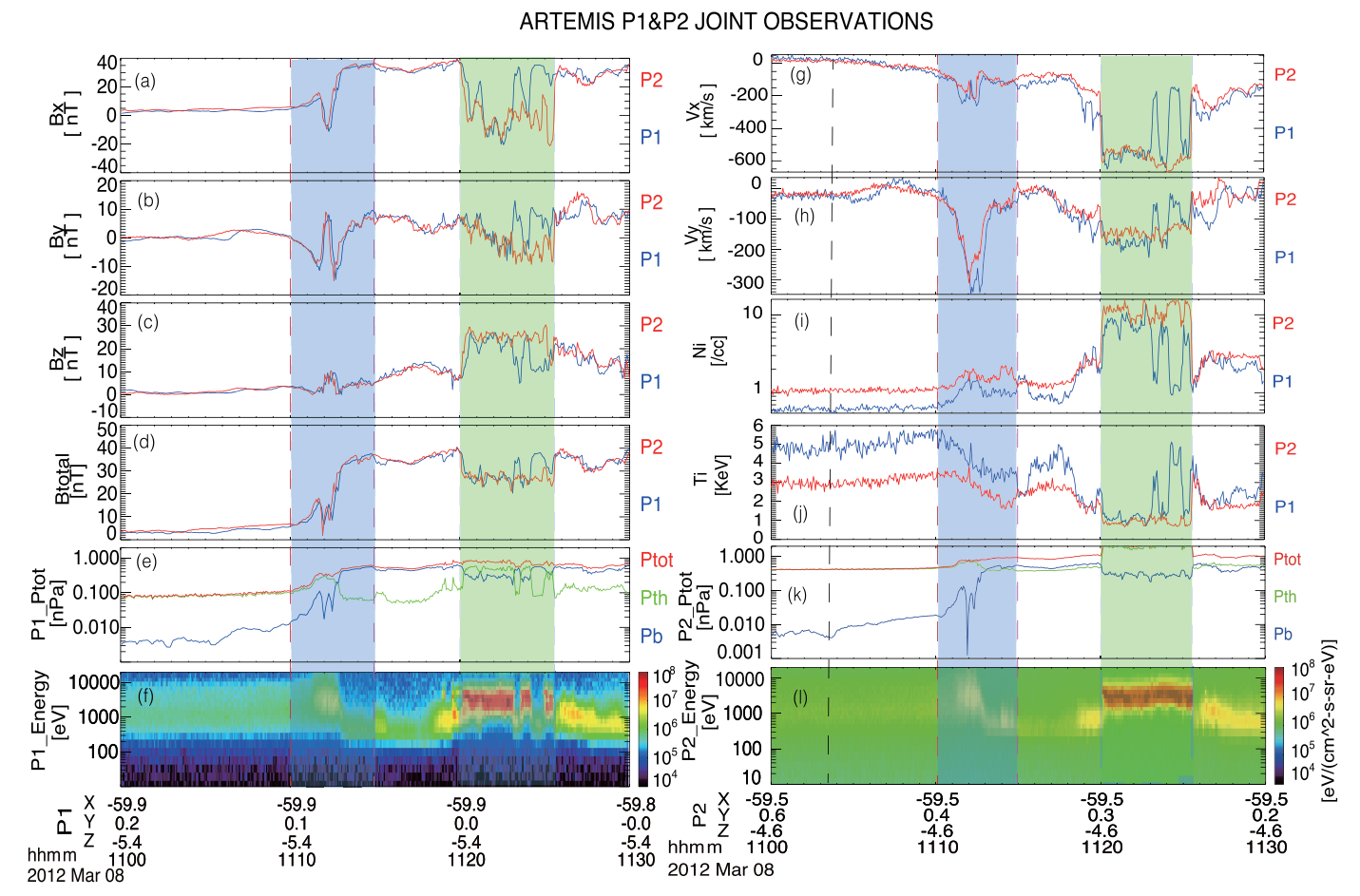

Figure 2. Magnetic field and plasma observations of P1 and P2 probes at the full moon time in the midnight region. In the top four panels, the (a) $B_{X}$, (b) $B_{Y}$, (c) $B_{Z}$, (d) magnetic field strength, (g) $V_{X}$, (h) $V_{Y}$, (i) ion density, and (j) ion average temperature from two different probes are plotted for comparison. In the bottom two panels, pressures and ion energy spectra from the P1 and P2 probes are shown separately in different panels. The magnetic pressures measured by two probes are shown in blue lines, thermal pressures in green lines, and total pressures in red lines. The arrival of the internal compression was seen by P2 probe marked by black dashed line at 11:03:30 UT. The external compression is marked by a blue shaded region that covers the beginning to the end of magnetic field magnitude increase.

multiple crossings of the magnetopause by the ARTEMIS probes during the 11:20 UT to 11:26 UT interval shown in Figure 2.

\section{Simulations}

Given the Wind, Cluster, ACE, and ARTEMIS probes observations described in the previous section that appear to indicate significant deformation in the deep magnetotail shape and location during the passage of an interplanetary (IP) shock, we conduct global MHD simulations to investigate how this occurs, and which solar wind and IP shock parameters play a dominant role in this case. In particular, we are interested in the roles played by the shock normal angle and solar wind $V_{Y}$ component in determining the magnetospheric response to the shock.

We use a global MHD simulation code developed by Hu et al. (2007) (which is an extension of the Lagrangian version of the PPM code Colella \& Woodward, 1984) to study the process observed by ARTEMIS probes. The solution to the MHD equations are given in GSE coordinates, with the simulation box extending from $X=$ $30 R_{E}$ to $-300 R_{E}$ along the $X$ axis, and from $-150 R_{E}$ to $150 R_{E}$ in the $Y$ and $Z$ directions, with $200 * 162 * 162$ grid points and a minimum grid spacing of $0.2 R_{E}$. More details of the simulation method can be found in Hu et al. (2007) and Tang and Wang (2018). The simulation is run under steady upstream solar wind and IMF conditions, which are based on the Wind, Cluster, and ACE satellites observations: the initial solar wind density is $10 \mathrm{~cm}^{-3}$, the dynamic pressure is $5 \mathrm{nPa}$, the solar wind velocity vector is $(-550,0,0) \mathrm{km} / \mathrm{s}$, the interplanetary magnetic field vector is $(-10.9,-1.7,8.1) \mathrm{nT}$, and the Earth's geomagnetic field dipole tilt angle is zero. The near-Earth inner boundary of the code at $3 R_{E}$ radial distance is handled by incorporating an electrostatic ionosphere with an assumed uniform Pederson conductivity. Hence, a three-dimensional simulation of the coupled solar wind-magnetosphere-ionosphere is constructed. A simple discontinuity in otherwise constant solar wind parameters is used to model the shock front. Behind the shock, the density, pressure, solar wind velocity, and magnetic field vectors sharply changed to $15 \mathrm{~cm}^{-3}, 15 \mathrm{nPa},(-750,-150,-50)$ 

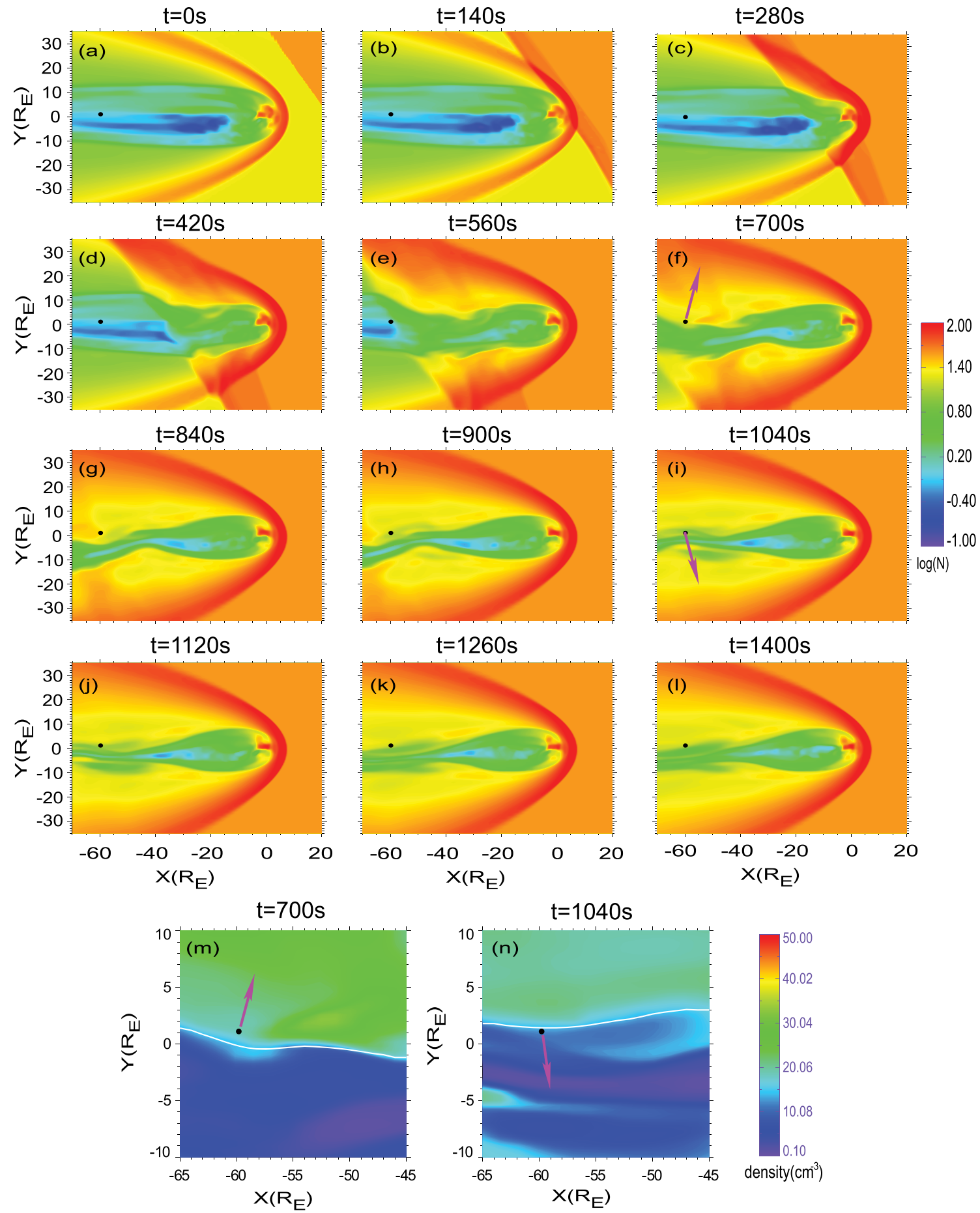

Figure 3. The X-Y distributions of density at $Z=-5.4 R_{E}$ plane at different time of the piecewise parabolic method (PPM) simulation in Geocentric Solar Ecliptic (GSE) coordinate. The black dot is the P1 probe location. The purple arrows are the normal of the magnetopause (marked by the white line in Panels ( $\mathrm{m}$ ) and (n)) or the relative trajectory of the probe in (f), (i), (m), and (n) panels. The color bar indicates plasma density. Panels (m) and (n) are the local parameters of the magnetohydrodynamic (MHD) simulation in the $X_{G S E}=[-65,-45] R_{E}$ and $Y_{G S E}=[-10,10] R_{E}$ rectangle. 


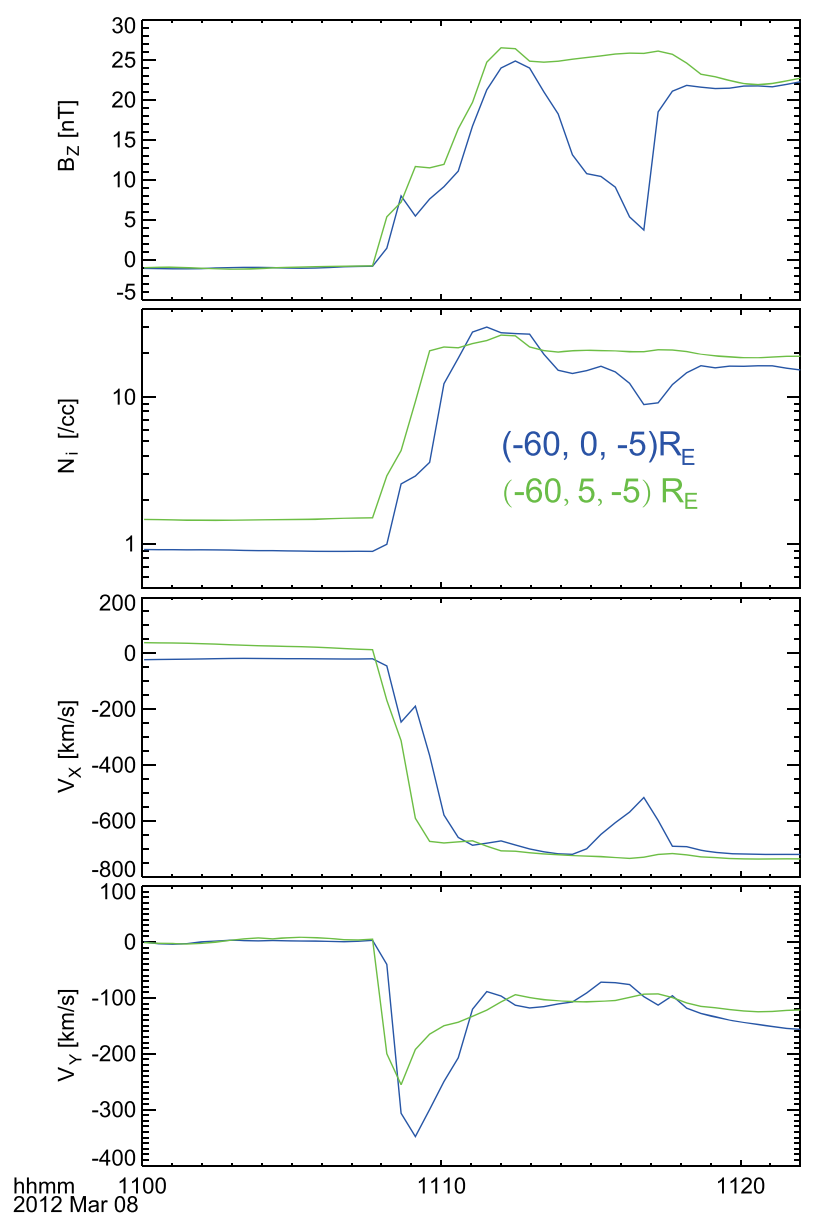

Figure 4. From top to bottom, the $B_{Z}$, ion density, $V_{X}$, and $V_{Y}$ parameters from two different locations of the piecewise parabolic method (PPM) simulation results. The blue lines represent the time series parameters of the virtual probe at $(-60,0,-5) R_{E}$, and the green lines represent the parameters at $(-60,5,-5) R_{E}$. $\mathrm{km} / \mathrm{s}$, and $(-26.8,-0.37,32.1) \mathrm{nT}$ in GSE coordinates, respectively. The normal of shock in the simulation set to $(-0.75,-0.47,-0.46)$.

Figure 3 shows the $X$ - $Y$ cross-section at $Z=-5.4 R_{E}$ of the simulated density for the simulation run. Plots (a-1) show a sequence of frames (with times indicated in each plot), starting prior to the shock arrival at the dayside magnetopause, in which the oblique shock front passes through near Earth interplanetary space. The shock front substantially compresses the magnetosheath and magnetosphere plasmas, causing antisunward propagating deformations in the magnotail after the shock passage. The black dot in each figure indicates the approximate ARTEMIS P1 location. The magnetopause boundary separates the magnetospheric plasma from the solar wind and is clearly visible in these plots, with magnetosheath plasma density significantly higher than that of the outer magnetosphere. Using these characteristics, we select the outer magnetosphere region as having the $N<10 \mathrm{~cm}^{-3}$, and the magnetosheath region as having the $N>10 \mathrm{~cm}^{-3}$ under the shock downstream conditions, shown in plots ( $\mathrm{m}$ and $\mathrm{n}$ ). Plot (a) shows that the geotail magnetopause location occurs at $Y=\sim 15 R_{E}$ (at $Z=-5.4 R_{E}$ plane at lunar distance) prior to the shock arrival. Plot (e) corresponds to the time at which the shock front arrives at the ARTEMIS P1 location, and (f) corresponds to its first magnetopause crossing. In order to distinguish the magnetopause and to easily identify it by eye, we present a detail of a local region $\left(-65 R_{E}<X<-45 R_{E} ;-10\right.$ $R_{E}<Z<+10 R_{E}$ ) in Figures $3 \mathrm{~m}$ and $3 \mathrm{n}$, correspond to Figures $3 \mathrm{f}$ and $3 \mathrm{i}$, respectively. In each of these plots, the arrows indicate the probe crossing direction with respect to the magnetopause (i.e., the opposite of the local normal direction of the magnetopause motion with respect to the probe).

The magnetopause is marked by a white line in these plots, based on the above criteria. As shown in Figure $3 \mathrm{f}$, at $t=700 \mathrm{~s}$, the magnetopause passes across the ARTEMIS P1 probe location antiparallel to the purple arrow direction (the direction approximately normal to the magnetopause, see more details in Figure $3 \mathrm{~m}$ ). Thereafter, the probe is completely in the magnetosheath, as shown in Figures $3 \mathrm{~g}$ and $3 \mathrm{~h}$. Subsequently, the magnetopause moves to its initial location antiparallel to the purple arrow direction at $t=1,040 \mathrm{~s}$ in Figure 3i (detailed in Figure 3n), such that the magnetopause boundary passes across the probe location again. After the magnetopause first crossed the probe, the magnetotail gradually tends to stabilize and tilts to $-Y$ direction, as shown in Figures $3 \mathrm{j}-31$. Ultimately, the ARTEMIS probe lies in the magnetosheath region towards the end of the simulation.

Figures $4 \mathrm{a}-4 \mathrm{~d}$ shows the time series of parameters $B_{Z}$, ion density, $V_{X}$, and $V_{Y}$ taken at two virtual probes (VP1 and VP2) located at $(-60,0,-5) R_{E}$ and $(-60,5,-5) R_{E}$, respectively, during the interval from 11:00 to 11:22 UT. The blue lines indicate that VP1 was in the magnetosphere from 11:00 to 11:08 UT. The green lines show that VP2 (located duskward of VP1) encountered the shock at 11:07:40 UT prior to VP1, then measured the compression effect from 11:08 to 11:09 UT. VP2 is continuously located within the magnetosheath region from 11:09:30 to 11:22 UT, while VP1 enters the magnetosheath region at 11:11 UT, then returns to the magnetosphere region at $\sim 11: 16$ UT (indicated by drops in ion density and $\left|V_{X}\right|$ ), before reentering the magnetosheath.

Increased magnetosheath pressure following the shock passage significantly reduces the radius of the magnetopause compared to its initial value in the simulation, as shown in Figure 3. However, not only is the magnetopause shape considerably compressed by the shock, but also the magnetopause location is deviated from its initial location in the simulation results. These results demonstrate that significant deviations in the magnetopause in the far magnetotail under the action of an obliquely directed shock front, such that a satellite at local midnight would experience multiple magnetopause crossings following the shock. 
We also ran a global MHD simulation through the Community Coordinated Modeling Center (CCMC) in which we used the OpenGGCM MHD code (Raeder et al., 2008) to run simulations of the shock-magnetosphere interaction. The input parameters are the same as the previous PPM simulation, except that the shock normal is $(-1,0,0)$ in the OpenGGCM simulation (i.e., the shock hits the magnetosphere straight towards the Earth) and the $B_{X}$ is a constant (we set $B_{X}=-10.9 \mathrm{nT}$ ), which are both different from the observation results and the input parameters in PPM MHD simulation. Therefore, the OpenGGCM simulation provides a point of reference to explore the effect of varying the shock normal angle. The interplanetary shock was introduced at 01:00 UT at $X=\sim 31 R_{E}$ well upstream of the bow shock (not shown here, see detail at http://ccmc.gsfc.nasa.gov). Then, the shock impacted the nose of magnetopause at $\sim 01: 03$ UT. After an 9-minute travel, the shock arrived at the lunar distance. The magnetosphere was sharply compressed within the lunar distance. At the same time, the magnetopause began to move toward the dawnside. At 01:16 UT, the magnetopause arrived at the midnight region at lunar orbit. Gradually, the magnetosphere tended to be stable after $\sim 01: 36 \mathrm{UT}$, which is confirmed by all the plasma parameters. The highest resolution of OpenGGCM simulation is $240 \mathrm{~s}$ so that we can not point out the details of magnetopause in response to the shock downstream conditions. However, the OpenGGCM simulation shows that magnetotail is deflected and the magnetosphere is considerably compressed at lunar distance, as before. From the OpenGGCM and another MHD simulation, we can obtain qualitatively similar results to the PPM simulations.

\section{Discussion}

Global MHD simulations described in the previous section were carried out to investigate motion in the tail magnetopause under the action of a passing shock front, in order to understand observations made by ARTEMIS P1 and P2 probes. As the IMF $B_{Y}$ becomes dominant, the magnetopause moves to lower latitude and becomes dawn-dusk asymmetric, and the tail cross-section also becomes distorted with the magnetopause shape elongating in the deep magnetotail (e.g., Lu et al., 2011; Sibeck \& Lin, 2014; Wang et al., 2014). We concentrate our attention in this discussion on the first outward and inward magnetopause crossings (occurring in the green shaded area of Figure 2). During this interval, the IMF $B_{Y}$ and dipole tilt angle (calculated to be $-3.5^{\circ}$ ) were weak and considered negligible for the purpose of the simulation. In order to aid our interpretation of the simulation data, a simplified shock front was considered, consisting of a single discontinuity separating otherwise constant IMF conditions, which were determined by average preshock and postshock IMF observations. Comparing the observations shown in Figure 2 with the simulations shown in Figure 3, it is remarkable that the ARTEMIS probes crossed the magnetopause at the full moon time in the midnight region. The shock first impacted the magnetopause at $\sim 11: 00 \mathrm{UT}$, then it arrived at the lunar orbit at $\sim 11: 10$ UT, hence the time delay is $\sim 10$ minutes. The corresponding time interval in the PPM simulation, from the shock arrival at the magnetopause $(t=85 \mathrm{~s})$ to the lunar orbit $(t=560 \mathrm{~s})$ at $(-60,0,-5) R_{E}$, is $\sim 8$ minutes in Figures 3 and 4.

As shown by the black dashed line of Figure 2, the disturbed magnetic field was first observed by P2 probe at 11:03:30 UT, and the shock impacted the magnetopause at 11:00 UT. According to the relative location between the first "shock-magnetopause tangent" region near the subsolar point of the Earth's magnetosphere and P1 probe $\left(\Delta \mathrm{X}=\sim 70 R_{E}\right)$, the velocity is $V=\Delta X / \Delta T=\sim 2000 \mathrm{~km} / \mathrm{s}$ inside the magnetosphere, which is much faster than the shock velocity in the solar wind. The disturbed magnetic field is mainly attributed to internal compressional waves in the magnetosphere (Zhou et al., 2013). Thereafter, the magnetic field sharply increased at 11:10 UT due to the external shock compression, which can be explained by considering pressure equilibrium as shown by the following equation:

$$
n_{p} V_{s w}^{2} \sin ^{2} \alpha+\frac{B_{s w}^{2}}{8 \pi}+P_{t h}=\frac{B_{L}^{2}}{8 \pi},
$$

where $n_{p}$ is the solar wind proton density, $\alpha$ is the tail flaring angle, $V_{s w}$ represents the solar wind velocity, $B_{s w}$ is the interplanetary magnetic field strength, $P_{t h}$ is the thermal pressure in the solar wind, and $B_{L}$ is the magnetic field strength in the tail lobe. The first term on the left-hand side is the perpendicular component of the solar wind dynamic pressure towards the tail magnetopause. The second and third terms on the left-hand side are the magnetic pressure and thermal pressure, respectively. Equation (1) shows that $B_{L}$ is determined by the external solar wind pressure, thermal pressure, and magnetic pressure. Thus, when the external shock arrived at the lunar distance at $~ 11: 10$ UT, the magnetic field increased abruptly. Based on five THEMIS probes' observations in $\sim 10-17 R_{E}$ in the tail, Zhou et al. (2013) have found two steps of compressions under 
Table 1

The P1 and P2 Data are Transformed Into a Local Boundary Coordinate System [L, M, N], Where L Contains the Main Magnetic Field Reversal and $N$ is the Boundary Normal

\begin{tabular}{lcccc}
\hline & \multicolumn{2}{c}{$11: 15: 00 \mathrm{UT}-11: 22: 00 \mathrm{UT}$} & \multicolumn{2}{c}{$11: 20: 00 \mathrm{UT}-11: 30: 00 \mathrm{UT}$} \\
& $\mathrm{P} 1$ & $\mathrm{P} 2$ & $\mathrm{P} 1$ & $\mathrm{P} 2$ \\
\hline$L$ & $(-0.93,-0.08,0.35)$ & $(-0.91,-0.08,0.42)$ & $(-0.94,-0.24,0.23)$ & $(-0.92,-0.33,0.20)$ \\
$M$ & $(0.35,-0.44,0.82)$ & $(0.38,-0.61,0.63)$ & $(0.22,0.04,0.97)$ & $(0.35,-0.47,0.81)$ \\
$N$ & $(0.09,0.89,0.44)$ & $(0.20,0.78,0.59)$ & $(0.24,-0.97,0.02)$ & $(0.17,-0.82,0.55)$ \\
$\left(\lambda_{1}, \lambda_{2}, \lambda_{3}\right)$ & $(229.5,-6.8,3.6)$ & $(300.6,5.2,3.0)$ & $(255.2,14.4,8.4)$ & $(343.2,18.8,14.9)$ \\
\hline
\end{tabular}

the neutral IMF $\left(B_{Z} \sim 0 \mathrm{nT}\right)$ precondition upstream of the shock. However, the event on 8 January 1998 has a strong northward IMF $B_{Z}$ precondition, and the Geotail satellite did not detect plasma sheet disturbance at (-28, - $\left.0.1,-3 R_{E}\right)$ GSM (Miyashita et al., 2010). In this paper, we find that the magnetotail central sheet at $-60 R_{E}$ also experienced a two-step transition from a quiescent to disturbed condition under the northward IMF precondition upstream of the shock. This is due to the compressional waves propagating faster in the tail than the shock propagates in the solar wind. However, for this specific shock event, we are not able to revisit the analysis in Zhou et al. (2013) due to lack of observations from near tail.

At $\sim 11: 13$ UT, the tail was thoroughly enveloped by the shock at lunar distance in the observations. The shock arrived at the lunar orbit at $t=560 \mathrm{~s}$ in Figure $3 \mathrm{e}$ in the simulation (i.e., 11:08 UT in Figure 4). The same result was supported by the OpenGGCM simulation. Due to the solar wind velocity vector deviation from the Sun-Earth line in the $Y$ and $Z$ directions and the shock effect, the P1 probe moved from the plasma sheet region to the lobe region at $\sim 11: 13$ UT. Before the first outward magnetopause crossing, the P1 probe was in the compressed lobe region from 11:13:00 UT to 11:20:00 UT. Compared with the plasma properties in the NS region between 11:00 UT and 11:10 UT, the lobe plasma with high density $\left(\sim 1 \mathrm{~cm}^{-3}\right)$ and low temperature $(\sim 3-4 \mathrm{KeV})$ is visible in Figure 2i during this period. In the simulation, however, ARTEMIS P1 was located in the compressed lobe region from $t=560$ to $700 \mathrm{~s}$ in the simulation (e.g., $t_{\text {lobe }}=140 \mathrm{~s}$, which is smaller than the observations). This may have resulted from the width of the ideal MHD discontinuity in the simulation, which is less than the Wind observations in Figures 1a-1f. In the absence of an oblique shock effect, the magnetopause moves slower toward dawnward in the OpenGGCM simulation than in the PPM simulation.

In order to determine the normal direction of the magnetopause surface as it crosses the spacecraft location, we use minimum variance analysis (MVA) based on the time series magnetic field data, see Table 1. During the interval 11:15:00-11:22:00 UT, just during the first inward magnetopause encounter, the P1 and P2 data are transformed into a local boundary coordinate system $[L, M, N]$ where $L$ contains the main magnetic field reversal and $N$ is the boundary normal. The $L, M$, and $N$ vectors correspond to maximum, intermediate, and minimum variance directions in Table 1 , respectively. This indicates that the normal of the magnetopause was $N_{P 1}=(0.09,0.89,0.44)$ as the P1 probe moved from the magnetosphere side into magnetosheath side. The P2 MVA results $\left(N_{P 2}=(0.20,0.78,0.59)\right)$ are consistent with the P1 MVA results. As expected from Figure $3 \mathrm{~m}$, the local normal points in the $(+X,+Y)$ direction (i.e., the magnetopause normal observed by $\mathrm{P} 1$ probe is consistent with the purple arrow direction). The normal of the magnetopause is consistent with the OpenGGCM simulation result. During the interval 11:22:00-11:30:00 UT, the P1 and P2 data are again transformed into a local boundary normal coordinate system. Figures $3 \mathrm{i}$ and $3 \mathrm{n}$ show the same results with the MVA results, we find that the P1 and P2 probes were crossed the magnetopause in the $(+X,-Y)$ direction from the magnetosheath side to the magnetosphere side during 11:22:00-11:30:00 UT. During the interval from Figures $3 \mathrm{f}$ to $3 \mathrm{i}, \Delta t=340 \mathrm{~s}$ in the simulation, which is almost the same as the observation result in the green shaded region of Figure 2. However, the process can't reproduced by the OpenGGCM simulation clearly, which may be caused by the time resolution of the simulation.

The flapping of the magnetotail in response to solar wind flow changes in Figure 3 is the windsock effect (Lundin et al., 2001; Shodhan et al., 1996; Vörös et al., 2014; Zong et al., 2004). The observation that the time scale for this effect at lunar distance is less than several hours is consistent with Geotail observations (Shodhan et al., 1996) taken at $-150 R_{E}$. As shown in Figures 3e-3k, the magnetic field disturbance is first observed by the virtual probe at $t=560 \mathrm{~s}$, and then the magnetosphere tends to a steady state by $t=1,260 \mathrm{~s}$, hence the flapping time is calculated $T_{f}=\Delta t=11.6$ minutes. The flapping time is $\sim 20$ minutes in the 


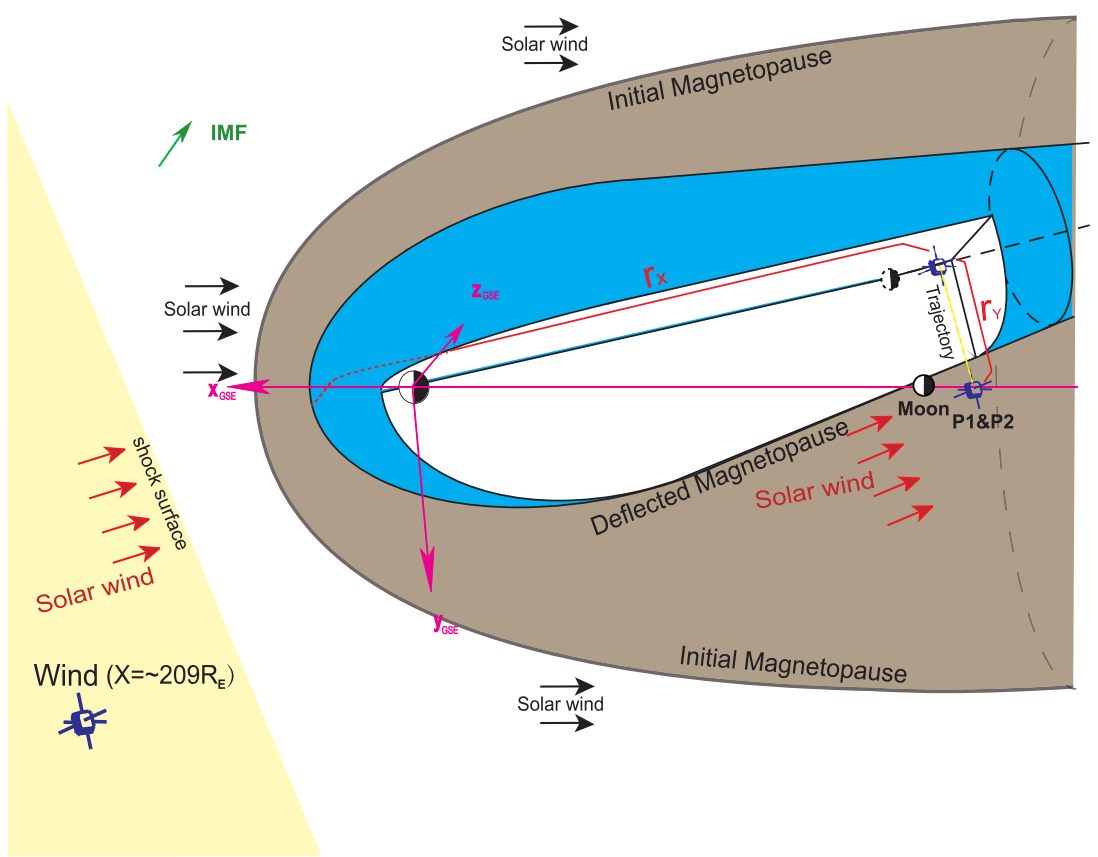

Figure 5. Three-dimensional cutaway view of Earth's magnetosphere, showing the compressed and deflected magnetopause in response to the shock arrival and solar wind velocity changes. The black and red arrows indicate the solar wind direction in the upstream and downstream of the shock, respectively. The gray shaded region is the initial magnetosphere location under the shock upstream condition. The blue shaded region represents the compressed and deflected magnetosphere after the shock arrival. The yellow dashed line indicates the relative trajectory of Acceleration, Reconnection, Turbulence, and Electrodynamics of the Moon's Interaction with the Sun (ARTEMIS) probes. After the shock arrival, the ARTEMIS probes and the moon crossed the magnetopause in the $+Y$ direction and thoroughly enveloped by the solar wind/magnetosheath flow at lunar distance near midnight (during the full moon time).

OpenGGCM simulation. The two simulations show that magnetotail is deflected and the magnetosphere is considerably compressed at lunar distance. It seems that the shocked tail at lunar distance is mainly controlled by the solar wind rather than the shock normal. According to the observations and simulations, we calculate that the time scale of the windsock effect is about a quarter hour at lunar distance in this event.

Figure 5 shows three-dimensional cutaway view of Earth's magnetosphere, showing the compressed and deflected magnetopause in response to the shock arrival and solar wind velocity changes in the blue shaded region. At the same time, the ARTEMIS probes and moon were thoroughly enveloped by the solar wind/magnetosheath flow at lunar distance near midnight. This means that the lunar nearside surface can be effectively impacted by the solar wind plasma during this period, shown in Figure 5. Figure 1 shows that not only did the $V_{X}$ component of the solar wind velocity change but also its dawnward component $\left(V_{Y}\right)$ deviated from the Sun-Earth line. Hence, as shown in Figures 3a and 5, we find that the ratio of the distance from the subsolar point of magnetopause to the P1 probe location $\left(r_{X}\right)$ and the flapping distance of the magnetopause in the $X-Y$ plane to the initial location of the magnetopause at lunar distance $\left(r_{Y}\right)$ is equal to the ratio of the velocity of downstream solar wind $V_{X}$ and $V_{Y}$ as follows:

$$
\frac{r_{X}}{r_{Y}}=\frac{V_{X}}{V_{Y}}
$$

This may indicate that $r_{Y}$ simply increases in response to increases in $V_{Y}$. However, the first term on the left-hand side in Equation (1) shows the dawnward solar wind has a great influence on the location of tail magnetopause. The solar wind dynamic pressure will increase at the same time. Thus, the radius of the magnetopause will decrease as $V_{Y}$ increases. If we only consider the solar wind $V_{Y}$ effect, the maximum deflection of the tail cross-section is about $20 R_{E}$, which is larger than the radius (about $15 R_{E}$ ) at $Z=-5.4$ $R_{E}$ plane at lunar distance under the shock upstream conditions. However, the velocity of flapping will be decreased without the oblique shock effect, which is confirmed by the OpenGGCM and PPM simulations. Although the compression effect of the shock has a great influence on the magnetopause location and shape 
in the magnetotail, it seems that the shocked tail at $60 R_{E}$ is mainly controlled by the solar wind velocity. The two MHD simulations show a qualitative agreement with the ARTEMIS observations. In addition, this case lays the foundation for a future magnetopause model which can include the effects of the solar wind $V_{Y}$. According to the observations and simulations mentioned above, the shocked magnetopause was observed by the P1 and P2 probes at the full moon time in the midnight region under an interplanetary shock downstream conditions. Hence, we can deduce that the windsock effect contributes to the deflection of the tail magnetopause, and the time scale is about a quarter hour at lunar distance in this case.

\section{Conclusions}

In order to study the location and shape of the magnetopause at lunar distance, we investigate the distributions of the plasma and magnetic field parameters observed by ARTEMIS probes. The ARTEMIS probes were near the Sun-Terrestrial line at the full moon time on 8 March 2012, where magnetosheath-like flows were observed by ARTEMIS probes many times within an hour after an oblique shock impacted the Earth magnetosphere. The solar wind velocity vector in the shock downstream was directed away from the Sun-Earth line, most strongly in the $Y$ direction. We use a global MHD simulation to reproduce the process of magnetopause crossings in the $X-Y$ plane under oblique shock conditions. The OpenGGCM simulation provides an additional reference to examine the effect of varying the shock normal angle.

Comparing the observations with simulation results, we find that the large-scale deflection of the magnetopause was observed by ARTMIES probes at the full moon time. Both global MHD simulations reproduced the crossing of the magnetopause at the full moon time in the region of $X=-60 R_{E}$ and $Y=0 R_{E}$. The PPM simulation results show a qualitative agreement with ARTEMIS observations. The MHD simulations provide a comprehensive global picture of the shocked tail that was significantly compressed and dragged dawnward by the solar wind. The shocked tail at $60 R_{E}$ is mainly controlled by the solar wind velocity. According to simple geometric considerations, the solar wind $V_{Y}$ is the dominant contribution to the deflection of the magnetopause with a timescale of less than half an hour at lunar distance, which is consistent with a windsock effect. The compression effect of the shock also has a significant influence on the magnetopause location and shape in the deep magnetotail in this event. The magnetotail central sheet at $60 R_{E}$ also experienced a two-step transition from a quiescent to a disturbed condition under the northward IMF $B_{Z}$ precondition in the shock upstream. This is due to the compressional waves propagating faster in the tail than the shock propagates in the solar wind. This case lays the foundation for a future magnetopause model which is expected to include the effects of the solar wind $V_{Y}$. The results also can provide some references for investigating the relationship between solar wind/magnetosheath and lunar nearside surface at the full moon time, which should not happen in general.

\section{References}

Akay, I. G., Kaymaz, Z., \& Sibeck, D. G. (2019). Magnetotail boundary crossings at lunar distances: ARTEMIS observations. Journal of Atmospheric and Solar-Terrestrial Physics, 182, 45-60. https://doi.org/10.1016/j.jastp.2018.11.002

Angelopoulos, V. (2011). The ARTEMIS Mission. Space Science Reviews, 165(1-4), 3-25. https://doi.org/10.1007/s11214-010-9687-2

Auster, H. U., Glassmeier, K. H., Magnes, W., Aydogar, O., Baumjohann, W., Constantinescu, D., et al. (2008). The THEMIS fluxgate magnetometer. Space Science Reviews, 141(1-4), 235-264. https://doi.org/10.1007/s11214-008-9365-9

Balogh, A., Carr, C. M., Acuña, M. H., Dunlop, M. W., Beek, T. J., Brown, P., et al. (2001). The Cluster Magnetic Field Investigation: Overview of in-flight performance and initial results. Annales Geophysicae, 19(10), 1207-1217. https://doi.org/10.5194/angeo-19-1207-2001

Baumjohann, W., Paschmann, G., \& Luehr, H. (1990). Characteristics of high-speed ion flows in the plasma sheet. Journal of Geophysical Research, 95(A4), 3801-3809. https://doi.org/10.1029/JA095iA04p03801

Chapman, S., \& Ferraro, V. C. A. (1931). A new theory of magnetic storms. Journal of Geophysical Research, 36(2), 77-97. https://doi.org/ 10.1029/TE036i002p00077

Colella, P., \& Woodward, P. R. (1984). The piecewise parabolic method (PPM) for gas-dynamical simulations. Journal of Computational Physics, 54, 174-201. https://doi.org/10.1016/0021-9991(84)90143-8

Coroniti, F. V., \& Kennel, C. F. (1972). Changes in magnetospheric configuration during the substorm growth phase. Journal of Geophysical Research, 77(19), 3361-3370. https://doi.org/10.1029/JA077i019p03361

Facskó, G., Honkonen, I., Živković, T., Palin, L., Kallio, E., Ågren, K., et al. (2016). One year in the Earth's magnetosphere: A global MHD simulation and spacecraft measurements. Space Weather, 14(5), 351-367. https://doi.org/10.1002/2015SW001355

Fairfield, D. H. (1971). Average and unusual locations of the Earth's magnetopause and bow shock. Journal of Geophysical Research, 76(28), 6700-6716. https://doi.org/10.1029/JA076i028p06700

Fairfield, D. H., Baumjohann, W., Paschmann, G., Lühr, H., \& Sibeck, D. G. (1990). Upstream pressure variations associated with the bow shock and their effects on the magnetosphere. Journal of Geophysical Research, 95(A4), 3773-3786. https://doi.org/10.1029/ JA095iA04p03773

Farrell, W. M., Thompson, R. F., Lepping, R. P., \& Byrnes, J. B. (1995). A method of calibrating magnetometers on a spinning spacecraft. IEEE Transactions on Magnetics, 31(2), 966-972. https://doi.org/10.1109/20.364770 
Gloeckler, G., Balsiger, H., Bürgi, A., Bochsler, P., Fisk, L. A., Galvin, A. B., et al. (1995). The solar wind and suprathermal ion composition investigation on the wind spacecraft. Space Science Reviews, 71(1-4), 79-124. https://doi.org/10.1007/BF00751327

Gordeev, E., Facskó, G., Sergeev, V., Honkonen, I., Palmroth, M., Janhunen, P., \& Milan, S. (2013). Verification of the GUMICS-4 global MHD code using empirical relationships. Journal of Geophysical Research: Space Physics, 118, 3138-3146. https://doi.org/10.1002/jgra 50359

Gou, X. C., Shi, Q. Q., Tian, A. M., Sun, W. J., Dunlop, M. W., Fu, S. Y., et al. (2016). Solar wind plasma entry observed by cluster in the high-latitude magnetospheric lobes. Journal of Geophysical Research: Space Physics, 121, 4135-4144. https://doi.org/10.1002/ 2015JA021578

Grygorov, K., Přech, L., Šafránková, J., Němeček, Z., \& Goncharov, O. (2014). The far magnetotail response to an interplanetary shock arrival. Planetary and Space Science, 103, 228-237. https://doi.org/10.1016/j.pss.2014.07.016

Harada, Y., Futaana, Y., Barabash, S., Wieser, M., Wurz, P., Bhardwaj, A., et al. (2014). Backscattered energetic neutral atoms from the moon in the earth's plasma sheet observed by Chandarayaan-1/Sub-keV atom reflecting analyzer instrument. Journal of Geophysical Research: Space Physics, 119, 3573-3584. https://doi.org/10.1002/2013JA019682

Harada, Y., Machida, S., Saito, Y., Yokota, S., Asamura, K., Nishino, M. N., et al. (2012). Nongyrotropic electron velocity distribution functions near the lunar surface. Journal of Geophysical Research, 117, A07220. https://doi.org/10.1029/2012JA017642

Hardy, D. A., Hills, H. K., \& Freeman, J. W. (1979). Occurrence of the lobe plasma at lunar distance. Journal of Geophysical Research, 84(A1), 72-78. https://doi.org/10.1029/JA084iA01p00072

Howe, J., Herbert, C., \& Binsack, J. H. (1972). Explorer 33 and 35 plasma observations of magnetosheath flow. Journal of Geophysical Research, 77(19), 3334-3344. https://doi.org/10.1029/JA077i019p03334

Hu, Y. Q., Guo, X. C., \& Wang, C. (2007). On the ionospheric and reconnection potentials of the earth: Results from global MHD simulations. Journal of Geophysical Research, 112(A7), A07215. https://doi.org/10.1029/2006JA012145

Kallio, E., \& Facskó, G. (2014). Properties of plasma near the moon in the magnetotail. Planetary and Space Science, 115, 69-76. https:// doi.org/10.1016/j.pss.2014.11.007

King, J. H., \& Papitashvili, N. E. (2005). Solar wind spatial scales in and comparisons of hourly wind and ace plasma and magnetic field data. Journal of Geophysical Research, 110(A2), A02104. https://doi.org/10.1029/2004JA010649

Liu, Z. Q., Lu, J. Y., Wang, C., Kabin, K., Zhao, J. S., Wang, M., et al. (2015). A three-dimensional high Mach number asymmetric magnetopause model from global MHD simulation. Journal of Geophysical Research: Space Physics, 120, 5645-5666. https://doi.org/10. 1002/2014JA020961

Liu, J., Shi, Q., Tian, A., Lü, J., Wu, H., Wang, M., et al. (2016). Shape and position of Earth's bow shock near-lunar orbit based on artemis data. Science China Earth Sciences, 59(8), 1700-1706. https://doi.org/10.1007/s11430-016-5319-3

Lu, J. Y., Liu, Z. Q., Kabin, K., Jing, H., Zhao, M. X., \& Wang, Y. (2013). The IMF dependence of the magnetopause from global MHD simulations. Journal of Geophysical Research: Space Physics, 118, 3113-3125. https://doi.org/10.1002/jgra.50324

Lu, J. Y., Liu, Z. Q., Kabin, K., Zhao, M. X., Liu, D. D., Zhou, Q., \& Xiao, Y. (2011). Three dimensional shape of the magnetopause: Global MHD results. Journal of Geophysical Research, 116(A9), A09237. https://doi.org/10.1029/2010JA016418

Lundin, R., Aparicio, B., \& Yamauchi, M. (2001). On the solar wind flow control of the polar cusp. Journal of Geophysical Research, 106(A7), 13,023-13,036. https://doi.org/10.1029/2000JA900021

McFadden, J. P., Carlson, C. W., Larson, D., Ludlam, M., Abiad, R., Elliott, B., et al. (2008). The THEMIS ESA plasma instrument and in-flight calibration. Space Science Reviews, 141(1-4), 277-302. https://doi.org/10.1007/s11214-008-9440-2

Miyashita, Y., Keika, K., Liou, K., Machida, S., Kamide, Y., Miyoshi, Y., et al. (2010). Plasma sheet changes caused by sudden enhancements of the solar wind pressure. Journal of Geophysical Research, 115(A5), A05214. https://doi.org/10.1029/2009JA014617

Ness, N. F., Behannon, K. W., Cantarano, S. C., \& Scearce, C. S. (1967). Observations of the Earth's magnetic tail and neutral sheet at 510,000 kilometers by Explorer 33. Journal of Geophysical Research, 72(3), 927-933. https://doi.org/10.1029/JZ072i003p00927

Nowada, M., Shue, J. H., \& Russell, C. T. (2009). Effects of dipole tilt angle on geomagnetic activity. Planetary Space Science, 57(11), 1254-1259. https://doi.org/10.1016/j.pss.2009.04.007

Opitz, A., Sauvaud, J.-A., Klassen, A., Gomez-Herrero, R., Bucik, R., Kistler, L. M., et al. (2014). Solar wind control of the terrestrial magnetotail as seen by STEREO. Journal of Geophysical Research, 119, 6342-6355. https://doi.org/10.1002/2014JA019988

Palmroth, M., Janhunen, P., Pulkkinen, T. I., \& Peterson, W. K. (2001). Cusp and magnetopause locations in global MHD simulation. Journal of Geophysical Research: Space Physics, 106(A12), 29,435-29,450. https://doi.org/10.1029/2001JA900132

Pan, D.-X., Sun, W., Shi, Q.-Q., Tian, A., Yao, Z., Fu, S.-Y., et al. (2016). THEMIS statistical study on the plasma properties of high-speed flows in Earth's magnetotail. Science China Earth Sciences, 59(3), 548-555. https://doi.org/10.1007/s11430-015-5199-y

Pan, D. X., Zhou, X.-Z., Shi, Q.-Q., Liu, J., Angelopoulos, V., Runov, A., et al. (2015). On the generation of magnetic dips ahead of advancing dipolarization fronts. Geophysical Research Letters, 42(11), 4256-4262. https://doi.org/10.1002/2015GL064369

Park, J.-S., Shue, J.-H., Kim, K.-H., Pi, G., Němeček, Z., \& A afránková, J. (2016). Global expansion of the dayside magnetopause for long-duration radial IMF events: Statistical study on GOES observations. Journal of Geophysical Research: Space Physics, 121, 6480-6492. https://doi.org/10.1002/2016JA022772

Pu, Z. Y., Zong, Q. G., Fritz, T. A., Xiao, C. J., Huang, Z. Y., Fu, S. Y., et al. (2005). Multiple flux rope events at the high-latitude magnetopause: Cluster/rapid observation on 26 January, 2001. Surveys in Geophysics, 26(1-3), 193-214. https://doi.org/10.1007/s10712-005-1878-0

Raeder, J., Larson, D., Li, W., Kepko, E. L., \& Fuller-Rowell, T. (2008). OpenGGCM simulations for the THEMIS mission. Space Science Reviews, 141(1-4), 535-555. https://doi.org/10.1007/s11214-008-9421-5

Reme, H., Aoustin, C., Bosqued, J. M., Dandouras, I., Lavraud, B., Sauvaud, J. A., et al. (2001). First multispacecraft ion measurements in and near the Earth's magnetosphere with the identical cluster ion spectrometry (CIS) experiment. Annales Geophysicae, 19(10/12), 1303-1354. https://doi.org/10.5194/angeo-19-1303-2001

Saito, Y., Yokota, S., Tanaka, T., Asamura, K., Nishino, M. N., Fujimoto, M., et al. (2008). Solar wind proton reflection at the lunar surface: Low energy ion measurement by MAP-PACE onboard SELENE (KAGUYA). Geophysical Research Letters, 35(24), L24205. https://doi. org/10.1029/2008GL036077

Shang, W., Yao, Z., Shi, Q., Sun, W., Fu, S., Liu, J., et al. (2014). Braking of high-speed flows in the magnetotail: THEMIS joint observations. Chinese Science Bulletin, 59(3), 326-334. https://doi.org/10.1007/s11434-013-0011-X

Shi, Q. Q., Hartinger, M., Angelopoulos, V., Zong, Q. G., Zhou, X. Z., Zhou, X. Y., et al. (2013). THEMIS observations of ULF wave excitation in the nightside plasma sheet during sudden impulse events. Journal of Geophysical Research: Space Physics, 118, 284-298. https://doi. org/10.1029/2012JA017984

Shi, Q. Q., Shen, C., Dunlop, M. W., Pu, Z. Y., Zong, Q. G., Liu, Z. X., et al. (2006). Motion of observed structures calculated from multi-point magnetic field measurements: Application to Cluster. Geophysical Research Letters, 33(8), L08109. https://doi.org/10.1029/ 2005GL025073 
Shi, Q. Q., Shen, C., Pu, Z. Y., Dunlop, M. W., Zong, Q. G., Zhang, H., et al. (2005). Dimensional analysis of observed structures using multipoint magnetic field measurements: Application to Cluster. Geophysical Research Letters, 32(12), L12105. https://doi.org/10.1029/ 2005GL022454

Shi, Q. Q., Tian, A. M., Bai, S. C., Hasegawa, H., Degeling, A. W., Pu, Z. Y., et al. (2019). Dimensionality, coordinate system and reference frame for analysis of in-situ space plasma and field data. Space Science Reviews, 215(4), 35. https://doi.org/10.1007/s11214-019-0601-2

Shi, Q. Q., Zong, Q. G., Fu, S. Y., Dunlop, M. W., Pu, Z. Y., Parks, G. K., et al. (2013). Solar wind entry into the high-latitude terrestrial magnetosphere during geomagnetically quiet times. Nature Communications, 4, 1466. https://doi.org/10.1038/ncomms2476

Shi, Q. Q., Zong, Q. G., Zhang, H., Pu, Z. Y., Fu, S. Y., Xie, L., et al. (2009). Cluster observations of the entry layer equatorward of the cusp under northward interplanetary magnetic field. Journal of Geophysical Research, 114(A12), A12219. https://doi.org/10.1029/ 2009JA014475

Shodhan, S., Siscoe, G. L., Frank, L. A., Ackerson, K. L., \& Paterson, W. R. (1996). Boundary oscillations at Geotail: Windsock, breathing, and wrenching. Journal of Geophysical Research, 101(A2), 2577-2586. https://doi.org/10.1029/95JA03379

Shue, J. H., Chao, J. K., Fu, H. C., Russell, C. T., Song, P., Khurana, K. K., \& Singer, H. J. (1997). A new functional form to study the solar wind control of the magnetopause size and shape. Journal of Geophysical Research, 102(A5), 9497-9512. https://doi.org/10.1029/97JA00196

Shue, J. H., Song, P., Russell, C. T., Steinberg, J. T., Chao, J. K., Zastenker, G., et al. (1998). Magnetopause location under extreme solar wind conditions. Journal of Geophysical Research, 103(A8), 17,691-17,700. https://doi.org/10.1029/98JA01103

Sibeck, D. G., Angelopoulos, V., Brain, D. A., Delory, G. T., Eastwood, J. P., Farrell, W. M., et al. (2011). ARTEMIS Science Objectives. Space Science Reviews, 165(1-4), 59-91. https://doi.org/10.1007/s11214-011-9777-9

Sibeck, D. G., \& Lin, R. Q. (2014). Size and shape of the distant magnetotail. Journal of Geophysical Research: Space Physics, 119, $1028-1043$. https://doi.org/10.1002/2013JA019471

Sibeck, D. G., Lopez, R. E., \& Roelof, E. C. (1991). Solar wind control of the magnetopause shape, location, and motion. Journal of Geophysical Research, 96(A4), 5489-5495. https://doi.org/10.1029/90JA02464

Song, P., \& Russell, C. T. (1992). Model of the formation of the low-latitude boundary layer for strongly northward interplanetary magnetic field. Journal of Geophysical Research, 97(A2), 1411-1420. https://doi.org/10.1029/91JA02377

Sun, W., Shi, Q., Fu, S., Zong, Q., Pu, Z., Xie, L., et al. (2010). Statistical research on the motion properties of the magnetotail current sheet: Cluster observations. Science China Technological Sciences, 53(6), 1732-1738. https://doi.org/10.1007/s11431-010-3153-y

Tang, B., \& Wang, C. (2018). Large scale current systems developed from substorm onset: Global MHD results. SCIENCE CHINA Technological Sciences, 61(3), 389-396. https://doi.org/10.1007/s11431-017-9132-y

Vörös, Z., Facskó, G., Khodachenko, M., Honkonen, I., Janhunen, P., \& Palmroth, M. (2014). Windsock memory COnditioned RAM (CO-RAM) pressure effect: Forced reconnection in the Earth's magnetotail. Journal of Geophysical Research: Space Physics, 119, 6273-6293. https://doi.org/10.1002/2014JA019857

Wang, C.-P., Lyons, L. R., \& Angelopoulos, V. (2014). Properties of low-latitude mantle plasma in the Earth's magnetotail: ARTEMIS observations and global MHD predictions. Journal of Geophysical Research: Space Physics, 119(9), 7264-7280. https://doi.org/10.1002/ 2014JA020060

Wang, H. Z., Zhang, J., Shi, Q. Q., Khurana, K. K., Rae, I. J., Liu, J., et al. (2018). Spatial variation of lunar surface hydration and the implications for its sources: Solar/earth winds or others. 49th Lunar and Planetary Science Conference 19-23 March, 2018, held at The Woodlands, Texas LPI Contribution No. 2083, id.2066.

Wang, H., Zhang, J., Shi, Q., Tian, A., Chen, J., Liu, J., et al. (2017). The possible influence of the Earth's magnetosphere on the formation of the lunar surface hydration. In Egu General Assembly Conference Abstracts, 19, pp. 10,936.

Wei, Y., Pu, Z., Zong, Q., Wan, W., Ren, Z., Fraenz, M., et al. (2014). Oxygen escape from the Earth during geomagnetic reversals: Implications to mass extinction. Earth and Planetary Science Letters, 394, 94-98. https://doi.org/10.1016/j.epsl.2014.03.018

Xiao, T., Shi, Q. Q., Zhang, T. L., Fu, S. Y., Li, L., Zong, Q. G., et al. (2010). Cluster-C1 observations on the geometrical structure of linear magnetic holes in the solar wind at 1 AU. Annales Geophysicae, 28(9), 1695-1702. https://doi.org/10.5194/angeo-28-1695-201

Yao, Z. H., Rae, I. J., Guo, R. L., Fazakerley, A. N., Owen, C. J., Nakamura, R., et al. (2017). A direct examination of the dynamics of dipolarization fronts using MMS. Journal of Geophysical Research: Space Physics, 122, 4335-4347. https://doi.org/10.1002/2016JA023401

Yao, S. T., Wang, X. G., Shi, Q. Q., Pitkänen, T., Hamrin, M., Yao, Z. H., et al. (2017). Observations of kinetic-size magnetic holes in the magnetosheath. Journal of Geophysical Research: Space Physics, 122, 1990-2000. https://doi.org/10.1002/2016JA023858

Zhang, X. Y., Zong, Q. G., Wang, Y. F., Zhang, H., Xie, L., Fu, S. Y., et al. (2010). ULF waves excited by negative/positive solar wind dynamic pressure impulses at geosynchronous orbit. Journal of Geophysical Research, 115(A10), A10221. https://doi.org/10.1029/2009JA015016

Zhou, X. Z., Angelopoulos, V., Runov, A., Sitnov, M. I., Coroniti, F., Pritchett, P., et al. (2009). Thin current sheet in the substorm late growth phase: Modeling of THEMIS observations. Journal of Geophysical Research: Space Physics, 114(A3), A03223. https://doi.org/10. 1029/2008JA013777

Zhou, X.-Y., Zhou, X.-Z., Angelopoulos, V., Shi, Q., Wang, C.-P., \& Frey, H. (2013). Interplanetary shock-induced current sheet disturbances leading to auroral activations: THEMIS observations. Journal of Geophysical Research: Space Physics, 118, 3173-3187. https://doi.org/10. 1002 /jgra. 50175

Zong, Q. G., Fritz, T. A., Zhang, H., Korth, A., Daly, P. W., Dunlop, M. W., et al. (2004). Triple cusps observed by Cluster-Temporal or spatial effect?. Geophysical Research Letters, 31(9), L09810. https://doi.org/10.1029/2003GL019128

Zong, Q. G., Wang, Y. F., Zhang, H., Fu, S. Y., Zhang, H., Wang, C. R., et al. (2012). Fast acceleration of inner magnetospheric hydrogen and oxygen ions by shock induced ULF waves. Journal of Geophysical Research, 117(A11), A11206. https://doi.org/10.1029/2012JA018024

Zong, Q. G., Wilken, B., Reeves, G. D., Daglis, I. A., Doke, T., Iyemori, T., et al. (1997). Geotail observations of energetic ion species and magnetic field in plasmoid-like structures in the course of an isolated substorm event. Journal of Geophysical Research, 102(A6), 11,409-11,428. https://doi.org/10.1029/97JA00076

Zong, Q. G., Zhou, X. Z., Wang, Y. F., Li, X., Song, P., Baker, D. N., et al. (2009). Energetic electron response to ULF waves induced by interplanetary shocks in the outer radiation belt. Journal of Geophysical Research, 114(A10), A10204. https://doi.org/10.1029/ 2009JA014393 